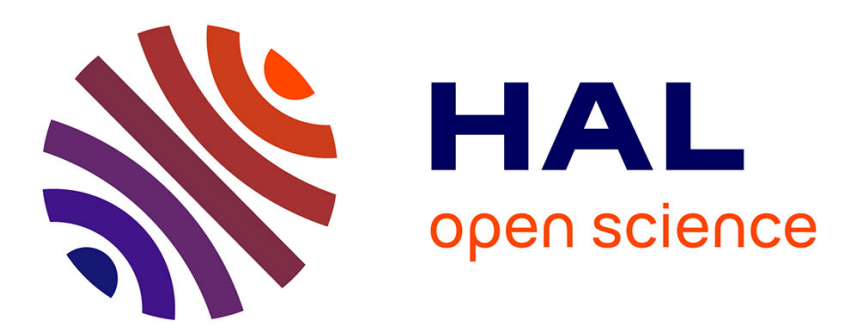

\title{
Transient Catalytic Voltammetry of Sulfite Oxidase Reveals Rate Limiting Conformational Changes
}

Ting Zeng, Silke Leimkuhler, Ulla Wollenberger, Vincent Fourmond

\section{To cite this version:}

Ting Zeng, Silke Leimkuhler, Ulla Wollenberger, Vincent Fourmond. Transient Catalytic Voltammetry of Sulfite Oxidase Reveals Rate Limiting Conformational Changes. Journal of the American Chemical Society, 2017, 139 (33), pp.11559-11567. 10.1021/jacs.7b05480 . hal-01614177

\section{HAL Id: hal-01614177 \\ https://hal-amu.archives-ouvertes.fr/hal-01614177}

Submitted on 16 Apr 2018

HAL is a multi-disciplinary open access archive for the deposit and dissemination of scientific research documents, whether they are published or not. The documents may come from teaching and research institutions in France or abroad, or from public or private research centers.
L'archive ouverte pluridisciplinaire HAL, est destinée au dépôt et à la diffusion de documents scientifiques de niveau recherche, publiés ou non, émanant des établissements d'enseignement et de recherche français ou étrangers, des laboratoires publics ou privés. 


\title{
Transient catalytic voltammetry of sulfite
} oxidase reveals rate limiting conformational

\section{changes}

\author{
Ting Zeng, ${ }^{\dagger}$ Silke Leimkühler, ${ }^{\dagger}$ Ulla Wollenberger, ${ }^{*,+}$ and Vincent Fourmond ${ }^{*, \ddagger}$ \\ IInstitute of Biochemistry and Biology, University of Potsdam, Karl-Liebknecht-Str.24-25, 14476 \\ Potsdam-Golm, Germany \\ $\ddagger$ Aix-Marseille Université, CNRS, BIP UMR 7281, 31 chemin J. Aiguier, F-13402 Marseille cedex \\ 20, France
}

E-mail: uwollen@uni-potsdam.de; vincent.fourmond@imm.cnrs.fr

\begin{abstract}
Sulfite oxidases are metalloenzymes that oxidize sulfite to sulfate at a molybdenum active site. In vertebrate sulfite oxidases, the electrons generated at the Mo center are transferred to an external electron acceptor via a heme domain, which can adopt two conformations: a "closed" conformation, suitable for internal electron transfer, and an "open" conformation suitable for intermolecular electron transfer. This conformational change is an integral part of the catalytic cycle. Sulfite oxidases have been wired to electrode surfaces, but their immobilization lead to a significant decrease in their catalytic activity, raising the question of the occurrence of the conformational change when the enzyme is on an electrode. We recorded and quantitatively modelled for the first time the transient response of the catalytic cycle of human sulfite oxidase immobilized on an electrode. We show that conformational changes still occur on the
\end{abstract}


electrode, but at a lower rate than in solution, which is the reason for the decrease in activity of sulfite oxidases upon immobilization.

\section{Introduction}

Sulfite oxidases (SO) are molybdenum enzymes ${ }^{1-5}$ that catalyze the oxidation of sulfite to sulfate ${ }^{6}$. They play a fundamental role in the degradation of sulfur-containing amino acids in animals, and their dysfunction in humans causes severe brain atrophy and death in early childhood ${ }^{7}$. In addition to the Mo cofactor at which the oxidation of sulfite occurs, vertebrate SO contain a single heme whose role is to transfer electrons from the active site to the redox partner. The crystal structure of chicken $\mathrm{SO}^{8}$ held a surprise in that the distance between the heme and the Mo site is $32 \AA$, much too large for electron transfer at a rate compatible with enzymatic turnover $\left(\approx 100 \mathrm{~s}^{-1}\right)$. This lead to the proposal that sulfite oxidase exists in two conformations, "open" and "closed". In the "open" conformation (observed in the crystal structure, figure 1), internal electron transfer is not possible, but the heme can react with external electron acceptors, whereas in the "closed" conformation, the heme domain is within tunneling distance of the Mo active site, but presumably unable to interact with external electron carriers ${ }^{9}$. Support for this hypothesis came from flash photolysis measurements of the rate of intramolecular electron transfer in the presence of viscogens, which demonstrated that the interdomain electron transfer rate depends on the solution viscosity in a way that suggests that a conformational change gates the electron $\operatorname{transfer}^{10}$.

Figure 1: Structure of the chicken sulfite oxidase (PDB $1 S O X^{8}$ ), with the mobile heme domain shown in orange, and the metallic cofactors (molybdenum active site and heme) shown as sticks.

Direct electrochemistry has been used for the last two decades to probe various as-

pects of the function of redox enzymes ${ }^{11-14}$. In this technique, the enzyme is directly 
and efficiently wired to an electrode that substitutes for the natural redox partner of the enzyme, and the electrical current provides an instant readout of the turnover frequency. Molybdenum enzymes such as DMSO reductase ${ }^{15}$, nitrate reductases ${ }^{16,17}$, and arsenite oxidase ${ }^{18}$ were among the first enzymes to be studied using direct electrochemistry. Sulfite oxidase and the closely related bacterial sulfite dehydrogenases have been immobilized on pyrolytic graphite edge electrodes ${ }^{19,20}$, gold electrodes modified with thiols ${ }^{21}$ or nanoparticles ${ }^{22-24}$, and silver electrodes ${ }^{25}$, either to gain mechanistic information, or in the perspective of constructing sulfite biosensors ${ }^{26}$ or the anodes of sulfite-based biofuel cells ${ }^{27}$.

In the first direct electrochemistry study of chicken $\mathrm{SO}(c \mathrm{SO})$, Elliott and coworkers could observe so-called "non-catalytic" signals ${ }^{19}$, in which the current results only from the oxidation and reduction of the heme $b_{5}$ redox cofactor, in the absence of turnover. These signals inform on the potential of the redox cofactors of the enzyme, and on the electroactive coverage, which must be known to measure absolute turnover rates from the value of the catalytic current. Elliott and coworkers remarked that the turnover rates of $c \mathrm{SO}$ on the electrode are significantly lower (about 20 times) than when the enzyme is in solution; this contrasts with the case of other molybdenum enzymes such as periplasmic (footnote 34 in ref 28) or respiratory nitrate reductase ${ }^{29}$, which are actually more active immobilized onto an electrode than in solution. This observation lead to the initial assessment that only a small fraction of the enzymes on the electrode (4\%) is in a configuration suitable for internal electron transfer, whereas the majority of the electroactive enzymes stays in a conformation that cannot turn over the substrate ${ }^{19}$. Alternatively, the conformational change may still occur on the electrode, but at a lower rate. Indeed, dipole moment calculations suggested that human $\mathrm{SO}(h \mathrm{SO})$ is immobilized on SAM-modified electrodes via its dimerization domain ${ }^{25}$, and molecular dynamics simulations suggested that $h \mathrm{SO}$ immobilized onto electrodes still undergoes conformational changes, albeit at a slower rate that strongly depends on buffer concentration ${ }^{30}$. 
The most simple way to learn about the catalytic properties of enzymes immobilized on electrodes is to analyze its response to linear sweeps of potential. The dependence of the steady-state catalytic current on potential, called the "wave shape", can be interpreted quantitatively to learn about various aspects of the catalytic cycle, provided it is modelled using a kinetic model that includes all relevant redox and non-redox steps ${ }^{31}$. This approach has been used successfully to study the binding of substrate ${ }^{17,32}$ or the presence of electronic relays ${ }^{13,33}$. In the case of small, non-catalytic proteins like ferredoxins, fast scan voltammetry ${ }^{34}$ has been used to learn about reactions coupled to electron transfers. The principle is that, by scanning the potential fast enough, one can outrun the reactions that follow or precede electron transfer. This has for instance been used to characterize coupled electron/proton transfers in ferredoxins ${ }^{35}$. However, this approach has been used only once with an enzyme under catalytic conditions ${ }^{36}$, and, to date, no framework is available for the quantitative analysis of the transient response of an enzymatic reaction to fast changes in electrode potential.

Here, we present the first quantitative modelling of fast scan voltammograms of a redox enzyme under turnover conditions. We have systematically recorded voltammograms under different conditions (scan rate and sulfite concentration), that range from "purely steady-state catalysis" to "purely non-catalytic" and especially at the transition between these two regimes, and we have compared the data with the predictions of various kinetic schemes. Only those that incorporate conformational changes for the intramolecular electron transfer in sulfite oxidase are compatible with the data. We conclude that these conformational changes still occur on the electrode, but slowed down to a point where they become rate-limiting. 


\section{Results}

\section{Experimental strategy}

The catalytic voltammograms of an enzyme immobilised on an electrode depend on the scan rate. In the limit of slow scan rates, in the presence of substrate, one expects to see a steady-state catalytic response: in the case of $h \mathrm{SO}$, it is plateau-shaped, and shows no hysteresis (the forward and backward responses are exactly the same, only offset by the capacitive current, see figure 2a). On the contrary, at very fast scan rates, one expects to outrun all coupled chemical reactions, in particular substrate turnover, and hence obtain a purely "non-catalytic" response. In the case of sulfite oxidase, the only non-catalytic signals recorded so far are those of the heme cofactor; no signals for the molybdenum center were observed ${ }^{19,25}$. Whether a voltammogram recorded under given conditions is at steady-state or not depends on $\kappa$, the ratio of the time scale of turnover $\left(\tau_{\text {cat }}=1 / k_{\text {cat }}\right.$, with $k_{\text {cat }}$ the turnover rate) over the time scale of voltammetry (the time it takes to sweep the potential across $R T / F, \tau_{\mathrm{CV}}=R T / F v$, in which $v$ is the scan rate $)^{37}$ :

$$
\kappa=\frac{\tau_{\mathrm{cat}}}{\tau_{\mathrm{CV}}}=\frac{F v}{R T k_{\mathrm{cat}}}
$$

At low values of $\kappa$, catalysis is much faster than the time scale of voltammetry, and the catalytic response is at steady-state. However, as $\kappa$ increases and becomes comparable to 1 or more, the response departs from the steady-state, and one can observe the transient response of the catalytic cycle. This occurs when $\kappa=1$, that is $v=v_{\text {trans }}$ :

$$
v_{\text {trans }}=\frac{R T k_{\mathrm{cat}}}{F}
$$

We used steady-state voltammograms at slow scan rate (figure 2a) to determine the turnover frequency $k_{\text {cat }}$ as a function of the concentration of sulfite (figure $2 \mathrm{~b}$ ). The plateau 

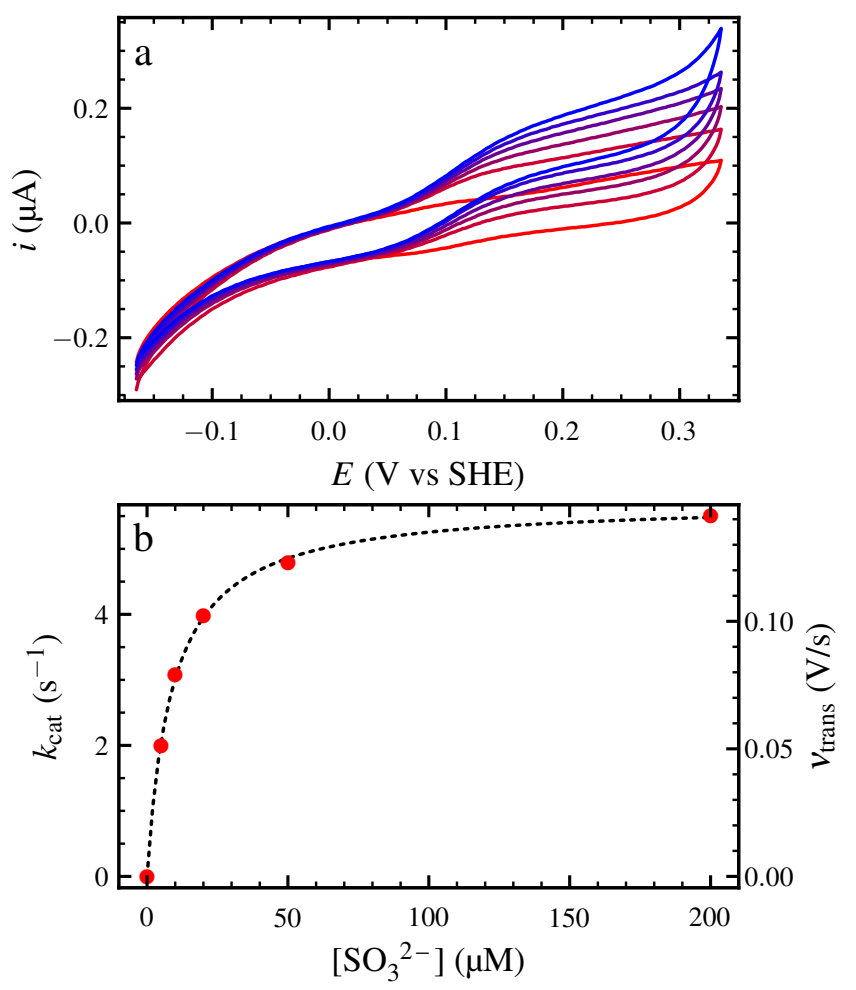

Figure 2: Panel a: cyclic voltammograms of films of $h \mathrm{SO}$ at $25 \mathrm{mV} / \mathrm{s}$ for increasing concentrations of sulfite. Panel b: red circles: catalytic rate constant determined from the plateau current of a film of immobilized sulfite oxidase for increasing concentrations of sulfite. The dashed line is a fit to Michaelis-Menten kinetics $k_{\mathrm{cat}}=k_{\mathrm{cat}}^{\max } /\left(1+K_{m} /\left[\mathrm{SO}_{3}{ }^{2-}\right]\right)$. The electroactive coverage was determined from the non-catalytic fits (see figure 6 and table 2). As $v_{\text {trans }}$ is proportional to $k_{\text {cat }}$ (eq (2)), one can also read $v_{\text {trans }}$ in panel $\mathrm{b}$ (right axis). Conditions: $h \mathrm{SO}$ immobilized on Au/DTSP / AuNP electrodes, $\mathrm{pH} 8.4, \mathrm{~T}=25^{\circ} \mathrm{C}$.

current is related to the catalytic rate by the following equation:

$$
i_{\text {plateau }}=n F \Gamma A k_{\mathrm{cat}}
$$

in which $\Gamma A$ is the electroactive coverage and $n$ is the number of electrons produced by the catalytic reaction (here, 2). $\Gamma A$ is generally determined from the value of the surface under the non-catalytic peaks; here, for the sake of consistency, we used the value of the electroactive coverage determined from the "non-catalytic" part of the fits to the data, see below (table 2). Sulfite oxidase displays a classical Michaelis-Menten behaviour, with $K_{m}=9 \pm 1 \mu \mathrm{M}$ and a maximum turnover rate of $k_{\mathrm{cat}}^{\max }=5.7 \pm 0.1 \mathrm{~s}^{-1}$, very similar to 
those previously determined for immobilized $h \mathrm{SO}\left(k_{\mathrm{cat}}^{\max }=3.17 \mathrm{~s}^{-1}, K_{m}=20 \mu \mathrm{M}\right.$ on CdS quantum dots ${ }^{23}, k_{\text {cat }}^{\max }=4.9 \mathrm{~s}^{-1}, K_{m}=6 \mu \mathrm{M}$ on gold nanoparticles ${ }^{24}$ ) and well below the catalytic rate of the enzyme in solution (in the order of $30 \mathrm{~s}^{-1}$ at $\mathrm{pH} 8.5$ with cytochrome $c$ as an acceptor $\left.{ }^{38,39}\right)$. This was also observed for $c \mathrm{SO}^{19,21}$. As $v_{\text {trans }}$ is proportional to the turnover frequency (equation (2)), we can also show it on the right axis in figure $2 b$. Its values are in the $0.1 \mathrm{~V} / \mathrm{s}$ range, greater than the scan rate used in figure 1 , so that $\kappa<1$.

To explore the whole range of behaviours, from voltammograms that are purely catalytic to ones those are almost purely non-catalytic, we have chosen to systematically record voltammograms with a series of sulfite concentrations ranging from 0 to 20 times $K_{m}$ and scan rates ranging between $25 \mathrm{mV} / \mathrm{s}$ (at most $\kappa=0.5$ ) to $4 \mathrm{~V} / \mathrm{s}$ (at least $\kappa=30$ ).

\section{The catalytic response of SO is not the superposition of independent catalytic and non-catalytic signals}

The low activity of the enzyme on the electrode with respect to that in solution was previously taken as an indication that the enzyme film is heterogeneous, with only a small fraction of the enzyme molecules contributing to catalysis, and most of the non-catalytic signal arising from catalytically inactive species ${ }^{19}$. If that hypothesis were true, the faradaic current would equate the sum of two independent terms:

$$
i=i_{\text {inact }}+i_{\text {act }}
$$

where $i_{\text {inact }}$ is the (non-catalytic) current of the catalytically inactive species, and $i_{\text {act }}$ is the contribution of the catalytically active species, which were assumed to give a steady-state response. As $i_{\text {inact }}$ does not depend on substrate concentration, subtracting the signal recorded in the absence of substrate from the catalytic signals should yield only $i_{\text {act }}$, which we expect has a sigmoidal shape (cf figure 1). Figure 3a shows two cyclic voltammograms recorded at the same scan rate $(170 \mathrm{mV} / \mathrm{s})$ : one in the absence of sulfite (black trace), and 
one at $200 \mu \mathrm{M}$ sulfite (red trace). Figure $3 \mathrm{~b}$ shows their difference. It is roughly sigmoidal in shape, but shows strong hysteresis, with a more intense current in the sweep towards low potentials than in the other direction. This is not compatible with it arising from a purely steady-state catalytic signal; the recorded signals therefore include transient relaxations of the catalytic cycle.

Limoges and Savéant derived an analytical solution for the current with an adsorbed 1-electron catalyst, under the assumption of fast electron transfer ${ }^{37}$. As these equations predict that the current is the sum of a purely non-catalytic signal and a purely steady-state signal, resulting in an equation for the current formally equivalent to (4), we cannot use these equations for our present work. An independent confirmation of that fact is that supplementary figure S5 shows that the assumption of a fast electron transfer is not verified here.

Elliott and coworkers proposed that the films of $c \mathrm{SO}$ were heterogeneous in order to resolve the apparent discrepancy between the non-catalytic signals and the catalytic, steady-state signals we recorded. Here, we show that the steady-state assumption does not hold, so the initial discrepancy is not relevant anymore. While we cannot conclude from figure $3 \mathrm{~b}$ alone that the film is homogeneous, the assumption of a heterogeneous film is no longer necessary to explain the results. For the sake of simplicity, we have therefore assumed in the rest of the article that the film is homogeneous. The good agreement between the data and the models demonstrates a posteriori that this assumption is justified.

\section{Modelling non-steady-state voltammograms}

To quantitatively model the data, one must first subtract a baseline to the raw voltammograms. We defined a baseline by fitting to the voltammograms recorded in the absence of sulfite two polynomial curves (one on either side of the non-catalytic peaks) connected by a straight line; we subtracted this baseline for all the voltammograms recorded at the same scan rate. This approach is illustrated in figure 4. 

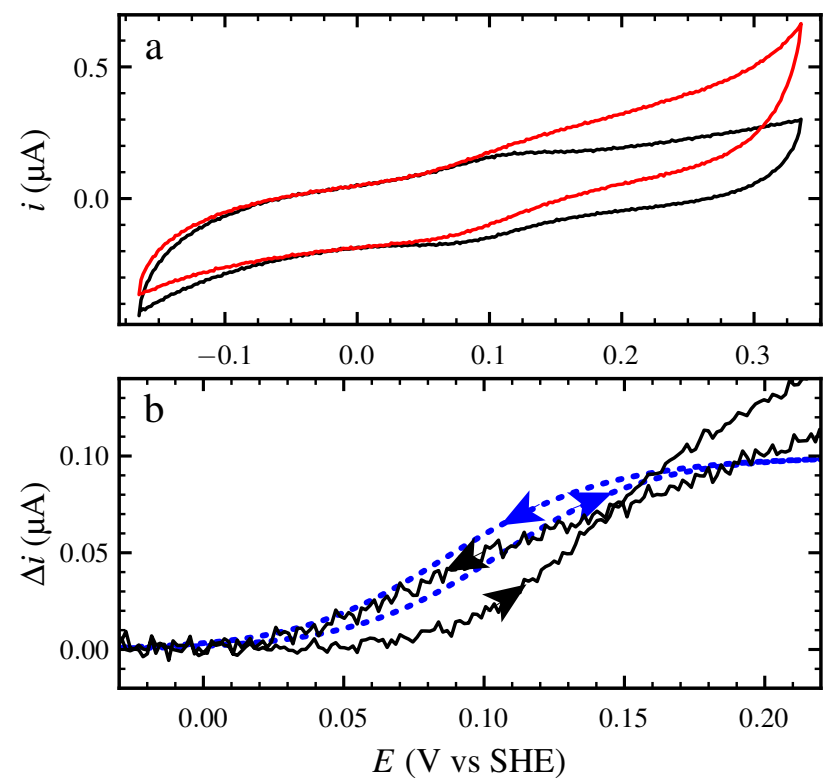

Figure 3: Panel a shows raw voltammograms of a film of immobilized sulfite oxidase at 0 (black trace) and $200 \mu \mathrm{M}$ sulfite (red trace). Panel b shows the difference between the voltammograms of panel a (black trace), together with the simulated difference from model c below (blue dotted line, see figure 6). Arrows indicate the directions of the sweeps. Experimental conditions: : $h \mathrm{SO}$ immobilized on Au/DTSP/AuNP electrodes, $\mathrm{pH} 8.4, \mathrm{~T}=$ $25^{\circ} \mathrm{C}, v=170 \mathrm{mV} / \mathrm{s}$, corresponding to $\kappa=1.2$.

To model the experimental voltammograms, we used three different kinetic schemes, which are represented in simplified form in figure 5. All of them feature two redox centers: the heme group (1-electron) and the molybdenum center (2-electron), and differ in the way the electrons are transferred between them. In model a, the electrons are irreversibly transferred from the molybdenum center to the heme. In model $b$, intramolecular electron transfer is reversible. Model c features irreversible electron transfer like in model a, but the enzyme exists under two conformations that interconvert: an open form, in which interfacial ET occurs, and a closed form in which irreversible intramolecular ET occurs. Models a and b have 6 microscopic states $(2$ redox states for the heme $\times 3$ redox states for the Mo), while model c has $12(2$ (heme) $\times 3(\mathrm{Mo}) \times 2$ (conformations)). All models include the following steps: reaction with sulfite (pseudo first-order rate $k_{s} \times\left[\mathrm{SO}_{3}{ }^{2-}\right]$ ), electron transfer from $\mathrm{Mo}(\mathrm{IV})$ to the heme (rate $k_{1}$ ), electron transfer from $\mathrm{Mo}(\mathrm{V})$ to the heme $\left(k_{2}\right)$, and reversible electron transfer from the heme to the electrode (rates $k_{f}$ and 

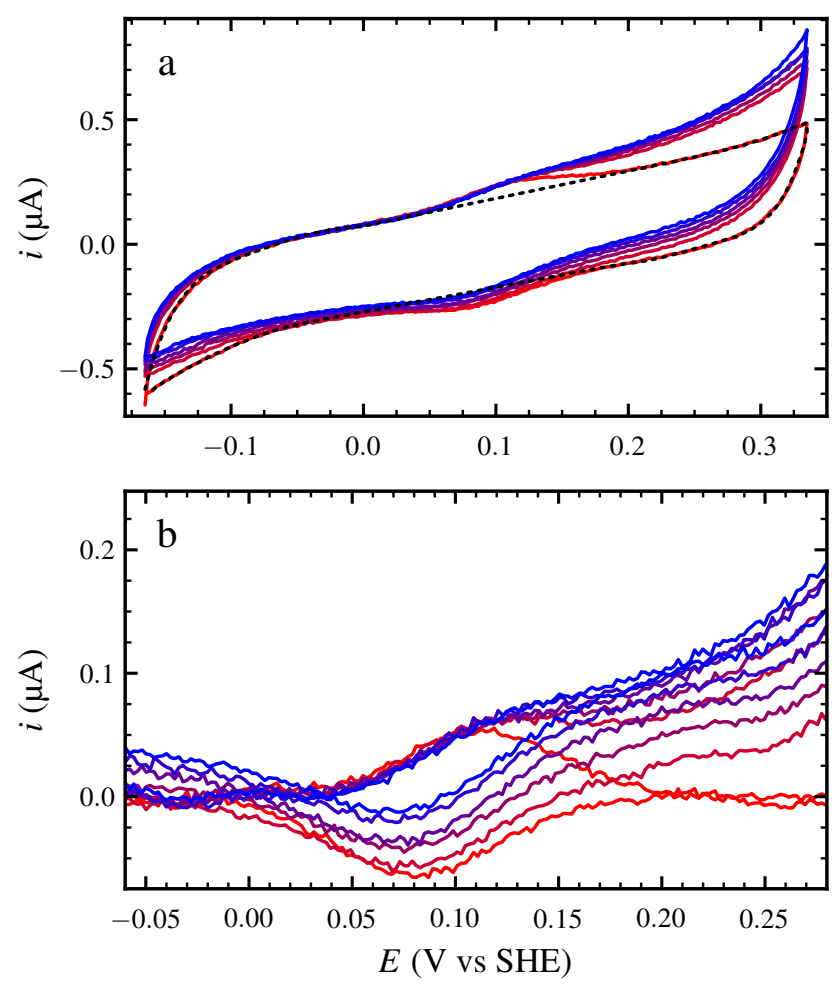

Figure 4: Panels a: raw cyclic voltammograms recorded at increasing concentrations of sulfite: 0 (red trace), 5, 10, 20, 50 and $200 \mu \mathrm{M}$ (blue trace), together with their baseline (black dashed lines). Panel b: baseline-subtracted data, with the same colors as in panel a. Conditions: : hSO immobilized on Au/DTSP/AuNP electrodes, $\mathrm{pH} \mathrm{8.4,} \mathrm{T}=25^{\circ} \mathrm{C}$, $v=300 \mathrm{mV} / \mathrm{s}$.

$k_{b}$, assumed to follow Butler-Volmer kinetics, equation (6) below). In addition, model b features the reverse reactions of the intramolecular electron transfers: from the reduced heme to the $\mathrm{Mo}(\mathrm{V})\left(\right.$ rate $k_{-1}$ ) and to the $\mathrm{Mo}(\mathrm{VI})$ (rate $k_{-2}$ ), and model c features the rate of the conformational change ( $k_{c}$ for closing and $k_{o}$ for opening).

The full details of the kinetic systems and the differential equations governing the evolution over time of the concentration of the species are given in SI section S1, together with the expression of the steady-state turnover rates. The differential equations are linear, but the presence of the Butler-Volmer terms, which strongly depend on potential (and hence on time), prevents any analytic integration ${ }^{40}$; we had therefore to resort to numerical 

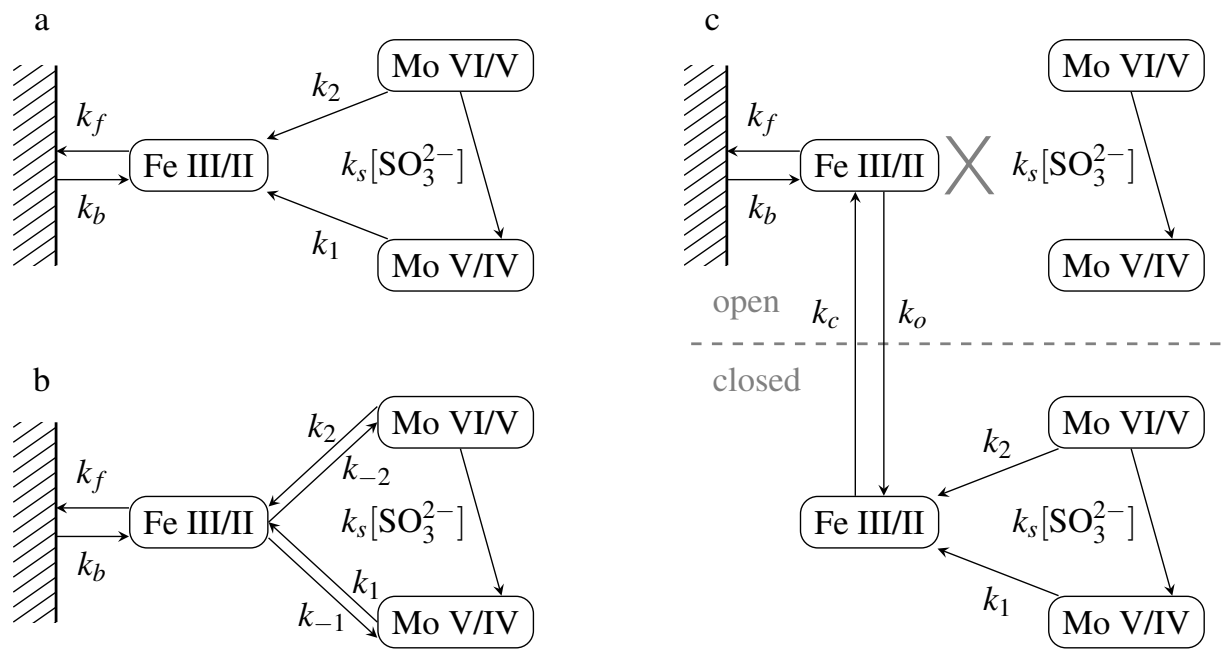

Figure 5: Schematic representation of the three kinetic schemes used for data fitting. Panels $a$ and $b$ depict the models where the Mo cofactor exchanges irreversibly (panel a) or reversibly (panel b) electrons with the heme relay, and conformational changes are not taken into account. Panel $c$ shows the case where interfacial electron transfer only occurs in the open conformation (top), whereas intramolecular ET occurs in the closed conformation; the two interconvert with 1 st order rate constants $k_{o}$ and $k_{c}$. Only the cofactors are represented, not the full microscopic states; the latter are depicted in supplementary figure S1.

integration. The current was determined using the following equation:

$$
i=n F A \Gamma\left(k_{f}[\mathrm{Fe}(\mathrm{II})]-k_{b}[\mathrm{Fe}(\mathrm{III})]\right)
$$

where $[\mathrm{Fe}(\mathrm{III})]$ (resp. [Fe(II)]) is the fraction of species (in the open conformation for model c) in which the heme is oxidized (resp. reduced), and $k_{f}$ and $k_{b}$ are given by:

$$
k_{f}=k_{0} \exp \frac{F\left(E-E_{0}\right)}{2 R T} \quad k_{b}=k_{0} \exp \frac{F\left(E_{0}-E\right)}{2 R T}
$$

where $E_{0}$ is the reduction potential of the heme, and $k_{0}$ the Butler-Volmer exchange rate constant.

For all the models, we used the same strategy for data fitting. We first determined the "non-catalytic parameters" (i.e the surface coverage $A \Gamma$, the reduction potential of the heme $E_{0}$ and $k_{0}$ ) by fitting all the non-catalytic data at once with a single set of parameters. 
Table 1: Potentials of the heme and the molybdenum center for various sulfite oxidases (literature data), together with the three possibilities for the equilibrium constants that were explored for fitting model $\mathrm{b}$ to the data.

\begin{tabular}{lcrrrrrll}
\hline Enzyme & $\mathrm{pH}$ & $E_{\text {heme }}(\mathrm{mV})$ & $E_{\mathrm{VI} / \mathrm{V}}(\mathrm{mV})$ & $k_{-2} / k_{2}$ & $E_{\mathrm{V} / \mathrm{IV}}(\mathrm{mV})$ & $k_{-1} / k_{1}$ & ref & \\
\hline human & 7.5 & 62 & 42 & 0.46 & & & 41 & $\begin{array}{l}\text { spectroelectrochemistry } \\
\text { human }\end{array}$ \\
\hline & 6 & & & 0.36 & & & 42 lash photolysis \\
\hline chicken & 6 & 90 & 131 & 4.9 & -86 & $1 \times 10^{-3}$ & 43 & microcoulometry \\
chicken & 9 & 51 & -57 & 0.015 & -233 & & 43 & microcoulometry \\
chicken & 7 & 68 & 38 & 0.31 & -239 & $6 \times 10^{-6}$ & 43 & microcoulometry \\
\hline Possibility 1 & 8.4 & & & 0.03 & & $10^{-1}$ & & \\
Possibility 2 & 8.4 & & & 0.03 & & $10^{-3}$ & & \\
Possibility 3 & 8.4 & & & 0.03 & & $10^{-5}$ & & \\
\hline
\end{tabular}

Then, we simultaneously fitted all the recorded voltammograms (all scan rates and all substrate concentrations, including 0), keeping the "non-catalytic parameters" fixed. This way, the fits are extremely constrained, since we can fit each model to a large number of catalytic datasets ( 9 scan rates $\times 6$ concentrations $=54$ voltammograms) by adjusting only a handful of parameters ( 2 for models a and b, 4 for model c, see table 2).

Despite the low number of parameters, we found that none of the models constrain well the rate constant $k_{1}$ of the oxidation of the Molybdenum (IV) by the heme $b_{5}$, so instead of letting this parameter free, we fitted the data three times for each model, assuming that (i) $k_{1}=k_{2}$, (ii) $k_{1}=10 \times k_{2}$, or (iii) $k_{1}=100 \times k_{2}$. Similarly, for model $\mathrm{b}$, the fits did not constrain the intramolecular electron transfer equilibrium constants. The potential of the heme is well known, and the equilibrium constant for the electron transfer between the heme and the $\mathrm{Mo}(\mathrm{VI}) / \mathrm{Mo}(\mathrm{V})$ couple has also been determined under various conditions using flash photolysis experiments, but as of now, the reduction potential for the $\mathrm{Mo}(\mathrm{V}) / \mathrm{Mo}(\mathrm{IV})$ couple is not known for $h \mathrm{SO}$ (it has been determined only for $c \mathrm{SO}$ ). We therefore considered three possibilities for the equilibrium constants of the intramolecular electron transfer reactions (table 1), based on available literature. Altogether, we performed 3 fits for each of model a and c (three values of the $k_{1} / k_{2}$ ratio), and 9 fits for model $\mathrm{b}$ (three values of $k_{1} / k_{2}$ combined with the three possibilities for the equilibrium constants). 
Table 2: Parameters of the fits of figure 6 . The first three parameters were adjusted only using non-catalytic data, as explained in text. Units: $E_{0}$ is in $\mathrm{mV}$ vs SHE, $F A \Gamma$ is in units of $10^{-8} \mathrm{C}, k_{0}, k_{2}, k_{0}$ and $k_{c}$ are in $\mathrm{s}^{-1}, k_{s}$ is in $\mathrm{s}^{-1} \cdot \mathrm{uM}^{-1}$, and $k_{1} / k_{2}, k_{1} / k_{-1}, k_{2} / k_{-2}$ are dimensionless. The error is the $95 \%$ confidence interval given by the fit engine.

\begin{tabular}{|c|c|c|c|c|c|c|c|c|c|c|}
\hline Model & $k_{0}$ & $E_{0}$ & $F A \Gamma$ & $k_{1} / k_{2}$ & $k_{2}$ & $k_{s}$ & $k_{0}$ & $k_{c}$ & $k_{1} / k_{-1}$ & $k_{2} / k_{-2}$ \\
\hline$a$ & $58 \pm 6$ & $95 \pm 2$ & $1.08 \pm 0.03$ & $\begin{array}{r}1 \\
10 \\
100\end{array}$ & $\begin{array}{r}10 \pm 3 \\
5 \pm 2 \\
6 \pm 2\end{array}$ & $\begin{array}{r}0.6 \pm 0.7 \\
0.4 \pm 0.4 \\
0.3 \pm 0.25\end{array}$ & & & & \\
\hline $\mathrm{b}$ & $58 \pm 6$ & $95 \pm 2$ & $1.08 \pm 0.03$ & $\begin{array}{r}1 \\
10 \\
100 \\
1 \\
10 \\
100 \\
1 \\
10 \\
100\end{array}$ & $\begin{array}{r}9 \pm 3 \\
4 \pm 2 \\
5 \pm 2 \\
9 \pm 3 \\
4 \pm 1.5 \\
5 \pm 2 \\
9 \pm 3 \\
4 \pm 1.5 \\
5 \pm 2\end{array}$ & $\begin{array}{r}0.9 \pm 1 \\
1 \pm 2 \\
0.5 \pm 0.6 \\
0.9 \pm 1 \\
1 \pm 2 \\
0.7 \pm 0.8 \\
0.9 \pm 1 \\
1 \pm 2 \\
0.7 \pm 0.8\end{array}$ & & & 0.03 & $10^{-3}$ \\
\hline c & $125 \pm 100$ & $96 \pm 2$ & $1.14 \pm 0.05$ & $\begin{array}{r}1 \\
10 \\
100\end{array}$ & $\begin{array}{r}300 \pm 70 \\
10^{6} \pm 10^{9} \\
10^{8} \pm 10^{11}\end{array}$ & $\begin{array}{l}0.49 \pm 0.02 \\
0.49 \pm 0.01 \\
0.49 \pm 0.01\end{array}$ & $\begin{array}{l}27 \pm 5 \\
25 \pm 1 \\
25 \pm 1\end{array}$ & $\begin{array}{r}16 \pm 4 \\
14.3 \pm 0.3 \\
14.3 \pm 0.3\end{array}$ & & \\
\hline
\end{tabular}

\section{Conformational changes still occur on the electrode}

The fits are shown together with the baseline-subtracted voltammograms in figure 6 , for the three different models (for $k_{1} / k_{2}=100$, and only one possibility for the equilibrium constants in the case of model b; all the fits are shown in figures S2 to S4). The fit parameters are given in table 2.

Globally, considering the very small number of free parameters for each of these fits ( 2 or 4 for 54 voltammograms), the fits are all very good regardless of the model, as they follow the trends visible in the experimental data: a sigmoidal shape with almost no hysteresis at low scan rates (left columns), and an almost purely non-catalytic signal at high scan rates (right columns).

The fits do not reproduce the increase in current at high potential, which is probably due to direct oxidation of sulfite (as it is present in the blanks in the presence of sulfite but in the absence of enzyme, supplementary figure S6), nor the drift of the baseline at low potential, which translates as an increase in current at high concentrations of sulfite in the low potential region.

However, a closer examination reveals that the fits of models a and b reproduce less 

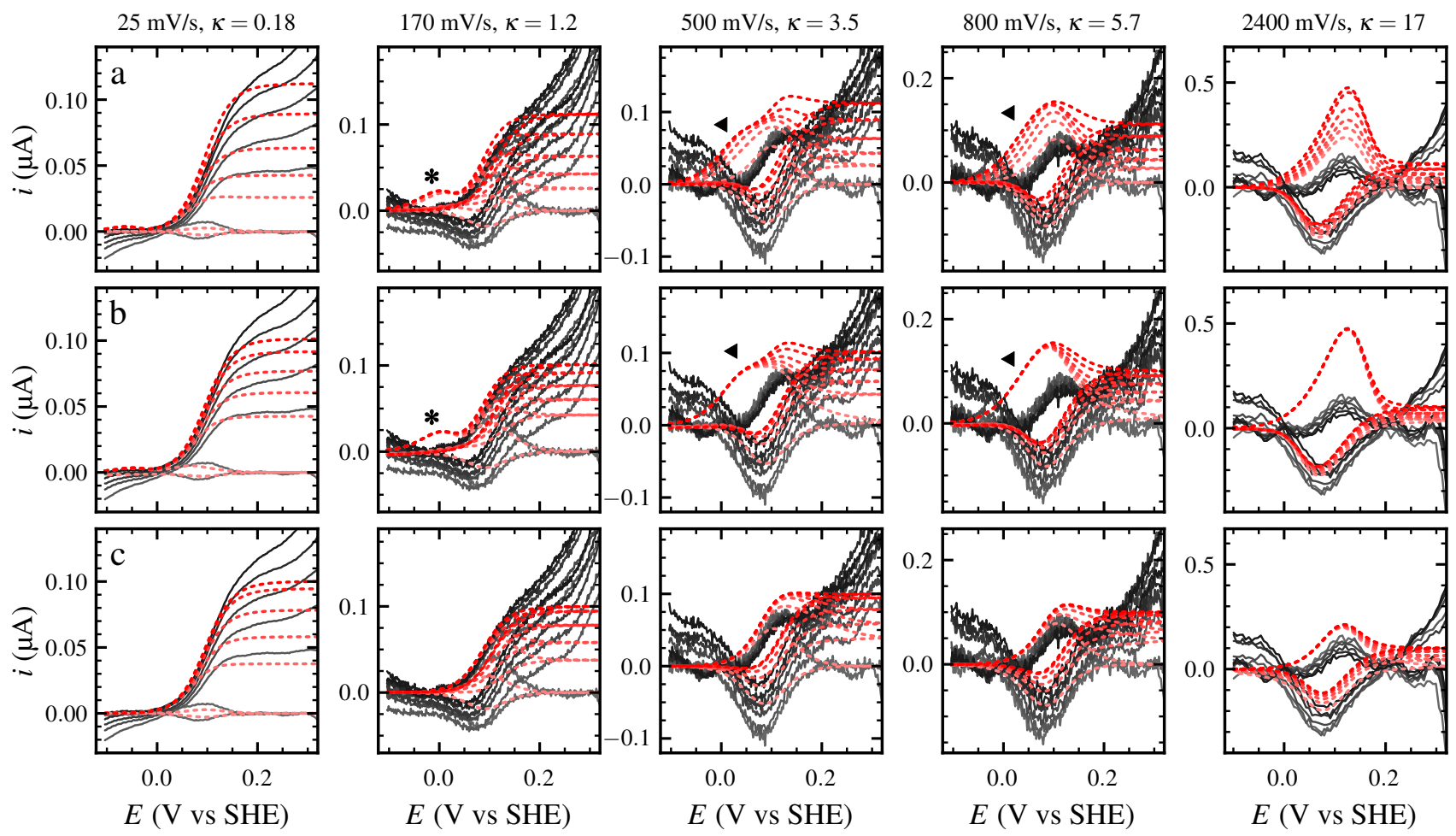

Figure 6: Baseline subtracted voltammograms (solid lines, at sulfite concentrations ranging from 0 , light gray to $200 \mu \mathrm{M}$, black), along with the fits of models $\mathrm{a}, \mathrm{b}$, and c (dashed lines) for $k_{1} / k_{2}=100$. The fits for all values of the $k_{1} / k_{2}$ ratio are shown in figures S2 to S4. The fit parameters are given in table 2. Conditions: $h \mathrm{SO}$ immobilized on Au/DTSP/AuNP electrodes, $\mathrm{pH} 8.4, \mathrm{~T}=25^{\circ} \mathrm{C}$, scan rates as indicated on the top of each column. The value of $\kappa$ indicated corresponds to the rate at the maximum concentration of sulfite.

well the features of the voltammograms than those of model c. For models a and b (1st and 2nd rows in fig 6), the current at fast scan rates in the upward direction is systematically overestimated, and the calculated peaks at intermediate scan rates are significantly wider than the experimental voltammograms, either starting at lower potentials than the data (arrows in figure 6) or even showing "pre-peaks" (indicated by asterisks) that are absent from the data. In contrast, the fits of model c reproduce much more faithfully the features of the experimental voltammograms, at the cost of 2 additional parameters. We conclude than model $\mathrm{c}$ reproduces better the data than models $\mathrm{a}$ and $\mathrm{b}$, and thus that the conformational change that plays an important role in the catalytic cycle in solution also takes place when the enzyme is on an electrode. 


\section{Discussion}

A simple and potentially very informative experiment to perform with redox enzymes immobilized on an electrode is to sweep the potential and record the current, hence the enzymatic activity, as a function of the driving force. The steady-state response, the socalled "wave-shape", has been modelled ${ }^{31,44}$ for different cases to learn for instance about the influence of additional inorganic cofactors that may serve as electron relays ${ }^{13,33}$ or the effect of slow binding of substrate ${ }^{17,32}$. Sometimes, this steady-state response of the catalytic cycle is modulated by slow inactivation/reactivation processes that take place over the duration of the voltammogram, which results in a departure from steady-state and marked hysteresis ${ }^{45,46}$. In this work, however, the departure from steady-state that we investigated does not reflect inactivation/reactivation processes, but arise from the fact that the electrode potential is swept fast enough to outrun the catalytic reaction, and the transients are relaxations of the catalytic cycle itself.

In this study of sulfite oxidase, we recorded a set of voltammograms under different conditions that span a large range of values of the number $\kappa$ (equation (1)) that quantifies the departure from steady-state. We note that the modest catalytic rate of immobilized sulfite oxidase is an asset for such experiments, because relatively small scan rates are required to outrun the catalytic reaction.

By subtracting the signals in the absence of sulfite from those in its presence (figure 3), we were able to show that the catalytic and non-catalytic contributions are not simply additive, and that the catalytic response is not at steady-state.

It is possible to derive analytical expressions of the steady-state current even if the interfacial electron transfer is assumed to be slow ${ }^{31}$, but it is only possible to integrate analytically the equations governing the transient response in current of a film of immobilized enzyme if the electron transfer is very fast ${ }^{37}$. As this was not the case here, we could not derive closed forms expressions for the current and had to resort to numerical integration to compute the theoretical curves. This was necessary since the curves that 
are the most discriminative with respect to the model are those with values of $\kappa$ close to one (figure 6), i.e. those that are neither "purely steady-state" nor "purely non-catalytic"; indeed, all models predict the same current responses at low and high values of $\kappa$.

Accurate baseline subtraction is essential to model the data. We used a simple method for computing baselines from the voltammograms in the absence of sulfite, and subtracted it from all the voltammograms at the same scan rate. However, the baseline evolves upon addition of sulfite, in a manner that is absent from control experiments in the absence of enzyme (figure S6). In particular at low potentials, the baseline tends to shift upwards upon increasing the sulfite concentration. This is unlikely to be a catalytic current, since the shift is much more pronounced for the reductive scan than for the oxidative scan, regardless of the scan rate. It could either result from traces of oxygen or perturbations of the double layer by the catalytic reaction.

We fitted three distinct models to the data, one in which there are no conformational changes, and electron transfer from the molybdenum is irreversible (model a), one in which electron transfer is reversible (model b) and conformational changes are not taken into account, and one in which electron transfer is irreversible, but only occurs when the enzyme is in a "closed" conformation which does not exchange electrons with the electrode (model c, figure 5). This corresponds to a situation in which the active site domain stays at a fixed position on the electrode, and the heme domain moves, acting as an electron shuttle between the electrode and the active site, as was predicted from theoretical computations ${ }^{30}$ (this configuration is illustrated in the graphical abstract). The other situation, in which the Mo domain moves while the heme domain stays in contact with the electrode closely resembles the model lacking conformational changes, at least when the enzyme is in the "closed" conformation; we have not considered this possibility further in our models. Out of the three models, the one that included conformational changes reproduced the features of the experimental data significantly better than the other two, which suggests that the conformational change, which is a critical step of the catalytic cycle in solution, still takes 
place on the electrode, albeit at a lower rate: the fits of model c give opening/closing rate constants of about 27 and $15 \mathrm{~s}^{-1}$, respectively (table 2), which corresponds roughly to a 2 to 1 equilibrium ratio in the favor of the open conformation. This model also yielded very high rate constants for the intramolecular electron transfers, with a very large 95\% confidence interval (see table 2). This reflects that the rate of intramolecular ET stricto sensu (i.e. the transfer in the closed conformation) is not rate-limiting. In fact, if one assumes that the conformational changes are the rate-limiting steps (at saturating sulfite concentrations), the 2 openings and 2 closings per catalytic cycle give the following expression for the maximum catalytic rate (see supplementary information section S1 for the derivation of steady-state rate constants):

$$
k_{\mathrm{cat}}^{\max }=\left(\frac{2}{k_{o}}+\frac{2}{k_{c}}\right)^{-1}=5 \pm 0.5 \mathrm{~s}^{-1}
$$

This value is similar to the maximum value of $k_{\text {cat }}$ deduced from the fit to the data in figure 2, which suggests that conformational change is indeed the rate-limiting step. This is consistent with the findings of Astashkin and coworkers that intramolecular electron transfer results in an obligatory conformational change ${ }^{47}$. Thus, at high scan rates, the voltammetry outruns the conformational changes and the electrons are never transferred between the heme and the molybdenum center. In this respect, it is interesting to note that, while all vertebrate sulfite oxidases studied so far only yield non-catalytic signals for the heme centers, Aguey-Zinsou and coworkers were able to record non-catalytic signals attributed to the molybdenum center in bacterial sulfite dehydrogenase ${ }^{20}$. Sulfite dehydrogenases are closely related to vertebrate sulfite oxidases, but their catalytic cycle does not involve any conformational change. Thus, the slowness of the conformational changes in vertebrate sulfite oxidase on the electrode is probably the reasons why no non-catalytic signals of the molybdenum center were ever recorded.

The rate of conformational change has never been directly measured in solution assays, 
but the rate of internal electron transfer between the heme and $\mathrm{Mo}(\mathrm{V})$ has been determined under various conditions both for chicken sulfite oxidase and human sulfite oxidase, using flash photolysis experiments. This rate most probably includes a conformational change, since its value decreases upon increasing solution viscosities ${ }^{10}$. Hence, in solution, the value of the slowest conformational rate constant must be at least as fast as intramolecular electron transfer, on the order of $400 \mathrm{~s}^{-1}$ for human sulfite oxidase at $\mathrm{pH} 7.5^{41}$. Consequently, the immobilization of the enzyme on the electrode leads to a decrease by at least an order of magnitude of the conformational change rate constant, most probably due to a lesser flexibility of the enzyme in the vicinity of the electrode. The values of the other rates constants determined from the fit (which are all significantly higher than the conformational change rate constant) suggest that the decrease in the rate of conformational change is the sole cause of the decrease in activity on the electrode with respect to the solution activity. In this sense, the nature of the electrode could play an important role, and it may be the reason why Ferapontova and coworkers ${ }^{21}$ obtained larger catalytic rates (about 4 times larger, to the point that the catalytic rates they measured were only a factor of 2 lower than the solution activity) than Elliott and coworkers ${ }^{19}$ despite using the same enzyme $(c \mathrm{SO})$. One of the possible explanations is that the SAM-covered gold electrode is softer than the pyrolytic graphite edge electrode, and hence allows for more flexibility of the immobilized enzyme and hence greater rates of conformational changes. This interpretation is also consistent with molecular dynamics simulations according to which the decrease in activity of immobilized enzymes in low buffer concentrations is due to a lesser flexibility of the enzyme on the electrode at low buffer concentrations ${ }^{30}$, and indeed, we found that increasing the solution viscosity decreases the catalytic activity of immobilized $h \mathrm{SO}^{24}$. 


\section{Conclusion}

We have explored systematically the transition between steady-state and transient catalytic cyclic voltammograms of immobilized $h \mathrm{SO}$. We were able for the first time to fit appropriate kinetic models to the resulting signals. Only models that include a conformational change could convincingly reproduce the electrochemical data, hence we conclude that the conformational change that is an integral part of the catalytic cycle in solution is also required with immobilized enzymes.

\section{Materials and methods}

Materials $\mathrm{Gold}(\mathrm{III})$-chloride trihydrate $\left(\mathrm{HAuCl}_{4} \cdot 3 \mathrm{H}_{2} \mathrm{O}\right)$ were provided by Aldrich. 3,3'-dithiodipropionic acid di (N-hydroxysuccinimide ester) (DTSP) and N-dodecyl-N,Ndimethylammonio-1-propanesulfonate were purchased from TCI. Branched polyethyleneimine (b-PEI, MW= $5000 \mathrm{~g} \cdot \mathrm{mol}^{-1}$ ) was from BASF. Potassium carbonate and heptanol was purchased from Fluka. Sulfuric acid was provided from Roth and nitric acid was from Merck. Goodfellow supplied platinum and gold wires with a diameter of $0.5 \mathrm{~mm}$. All chemicals were of analytical grade and used as received. All the solutions were prepared in $18 \mathrm{M} \Omega$ Millipore water.

Enzyme preparation His6-tagged human sulfite oxidase $(h \mathrm{SO})$ was purified after expression in E. coli TP1000 cells containing plasmid pTG718, as described previously ${ }^{48,49}$.

AuNP synthesis Synthesis of PEI capped AuNP in aqueous solution is according to the protocol reported ${ }^{50}$. An aqueous solution of PEI $(1 \mathrm{wt} \%)$ and $\mathrm{HAuCl}_{4}(2 \mathrm{mM})$ were mixed in a weight ratio PEI:HAuCl$=1: 1$ at room temperature and then heated up to $100^{\circ} \mathrm{C}$ for $5 \mathrm{~min}$. After cooling down to room temperature in a water bath, the red color solution indicates the formation of a colloidal AuNP dispersion. The nanoparticles solution was 
kept for storage at $4^{\circ} \mathrm{C}$ in the fridge.

Electrode modification The gold electrodes were cleaned as described ${ }^{22}$. Briefly, gold wires were cleaned in boiling $2 \mathrm{M} \mathrm{KOH}$ for $4 \mathrm{~h}$ and in concentrated $\mathrm{HNO}_{3}$ for $10 \mathrm{~min}$ with careful rinsing with Millipore water after every step. The electrodes were stored in concentrated $\mathrm{H}_{2} \mathrm{SO}_{4}$ when not in use. The surface was modified with the following procedures $^{24}$. The cleaned electrodes were immersed for $2 \mathrm{~h}$ into a $50 \mathrm{mM}$ solution of 3,3'-dithiodipropionic acid di(N-hydroxysuccinimide ester) (DTSP) in DMSO at room temperature. The DTSP modified electrodes were then rinsed with DMSO, water and then immersed in $0.2 \mathrm{M} \mathrm{K}_{2} \mathrm{CO}_{3} \mathrm{pH} 10.0$ for 20 minutes in order to activate the DTSP. The activated electrodes were incubated in AuNP nanoparticles solution for $1 \mathrm{~h}$ at room temperature. For attachment of $h \mathrm{SO}$ the nanoparticles modified gold electrodes (Au/DTSP/AuNP) were dipped in a $2 \mu \mathrm{M} \mathrm{hSO}$ solution ( $0.5 \mathrm{mM}$ Tris buffer, at $\mathrm{pH} 7.0)$ for $30 \mathrm{~min}$ at $4^{\circ} \mathrm{C}$. Modified electrodes were stored dry at $4^{\circ} \mathrm{C}$ when not in use. Prior measurement, the prepared electrodes were thoroughly rinsed with $5 \mathrm{mM}$ Tris-acetate solution $\mathrm{pH} 8.4$ to remove not tightly bound enzyme. The Au/DTSP/AuNP blank electrodes were prepared following the same procedure, but omitting of $h \mathrm{SO}$.

Experiment process Electrochemical measurements were carried out using a custommade $1 \mathrm{~mL}$ measuring chamber equipped with an $\mathrm{Ag} / \mathrm{AgCl} / 1 \mathrm{M} \mathrm{KCl}$ reference electrode $(E=+235 \mathrm{mV}$ vs standard hydrogen electrode) and a platinum wire counter electrode. The working electrodes were the Au-wires modified according to the procedure described above. Voltammetric measurements were performed at room temperature with the $\mu$ Autolab Type III/FRA2 potentiostat from Metrohm Autolab B.V. Cyclic voltammograms of modified Au/DTSP/AuNP/hSO electrode were recorded at nine different scan rates $(25,100,170,300,500,800,1400,2400,4000 \mathrm{mV} \mathrm{s}-1)$ in a $750 \mathrm{mM}$ Tris-acetate buffer solution at $\mathrm{pH}$ 8.4. After a series of measurements for all the scan rates, different concentrations of sulfite $(0,5,10,20,50$ and $200 \mu \mathrm{M})$ were added into Tris-acetate buffer 
solution. For each sulfite concentration, the same series of measurements at various scan rates were repeated again. The series of all the experiments were done in both aerobic and anaerobic condition. For the blank experiment all the measurement were repeated at $\mathrm{Au} / \mathrm{DTSP} / \mathrm{hSO}$ electrode as well. We used a stationary electrode to minimize electrical noise. The plateau shape of the voltammograms recorded at the lowest scan rates show that there is no depletion of sulfite. For all voltammograms, we recorded three cycles, and showed and modeled only the last scan.

Data processing and modelling We used QSoas ${ }^{51}$ for all data analysis. For the baseline subtraction, we used the two-polynomials fit setting a large error (hence a low weight) in the region of the data where the non-catalytic peaks were present. For the modelling, we used the ODE integration fits with the implicit Bulirsch-Stoer solver of Bader and

Deuflhard ${ }^{52}$ (QSoas solver bsimp), that greatly outperformed the other solvers available. To ensure that the models reproduced correctly the steady-state response of the enzyme, we averaged the forward and backward scans of the $v=25 \mathrm{mV} / \mathrm{s}$ voltammograms recorded in the presence of sulfite and gave them 5 times more weight than the other voltammograms.

\section{Acknowledgement}

The authors thank Christophe Léger for critical reading of the manuscript. We thank Joachim Koetz for synthesis of nanoparticles. VF acknowledges support from CNRS, Agence Nationale de la Recherche (ANR-12-BS08-0014, ANR-14-CE05-0010) and the A*MIDEX project ( ${ }^{\circ}$ ANR-11-IDEX-0001-02) funded by the «Investissements d'Avenir» French Government program, managed by the French National Research Agency (ANR). VF is a member of the French Bioinorganic Chemistry group (http://frenchbic.cnrs.fr). UW and SL acknowledge support from the Deutsche Forschungsgemeinschaft (Unicat Cluster of Excellence, EXC 314). 


\section{Supporting Information Available}

Supplementary figures S1 to S6, the differential equations used for data modelling, and the steady-state rates for the models.

\section{References}

(1) Hille, R.; Hall, J.; Basu, P. Chem. Rev. 2014, 114, 3963-4038.

(2) Pushie, M. J.; Cotelesage, J. J.; George, G. N. Metallomics 2014, 6, 15-24.

(3) Grimaldi, S.; Schoepp-Cothenet, B.; Ceccaldi, P.; Guigliarelli, B.; Magalon, A. Biochim. Biophys. Acta 2013, 1827, 1048-1085.

(4) Iobbi-Nivol, C.; Leimkühler, S. Biochim. Biophys. Acta 2013, 1827, 1086-1101.

(5) Schwarz, G.; Mendel, R. R.; Ribbe, M. W. Nature 2009, 460, 839-847.

(6) Feng, C.; Tollin, G.; Enemark, J. H. Biochim. Biophys. Acta 2007, 1774, 527-539.

(7) Schwahn, B. C.; Van Spronsen, F. J.; Belaidi, A. A.; Bowhay, S.; Christodoulou, J.; Derks, T. G.; Hennermann, J. B.; Jameson, E.; König, K.; McGregor, T. L.; FontMontgomery, E.; Santamaria-Araujo, J. A.; Santra, S.; Vaidya, M.; Vierzig, A.; Wassmer, E.; Weis, I.; Wong, F. Y.; Veldman, A.; Schwarz, G. The Lancet 2015, 386, 1955-1963.

(8) Kisker, C.; Schindelin, H.; Pacheco, A.; Wehbi, W. A.; Garrett, R. M.; Rajagopalan, K. V.; Enemark, J. H.; Rees, D. C. Cell 1997, 91, 973-983.

(9) Pacheco, A.; Hazzard, J. T.; Tollin, G.; Enemark, J. H. J. Biol. Inorg. Chem. 1999, 4, 390-401.

(10) Feng, C.; Kedia, R. V.; Hazzard, J. T.; Hurley, J. K.; Tollin, G.; Enemark, J. H. Biochemistry 2002, 41, 5816-5821. 
(11) Armstrong, F. A.; Heering, H. A.; Hirst, J. Chem. Soc. Rev. 1997, 26, 169-179.

(12) Léger, C.; Bertrand, P. Chem. Rev. 2008, 108, 2379-2438.

(13) Fourmond, V.; Baffert, C.; Sybirna, K.; Lautier, T.; Abou Hamdan, A.; Dementin, S.; Soucaille, P.; Meynial-Salles, I.; Bottin, H.; Léger, C. J. Am. Chem. Soc. 2013, 135, 3926-3938.

(14) Fourmond, V.; Léger, C. Adv. Biochem. Eng. Biotechnol. 2016, (advanced online publication), $1-41$.

(15) Heffron, K.; Léger, C.; Rothery, R.; Weiner, J.; Armstrong, F. Biochemistry 2001, 40, $3117-3126$.

(16) Anderson, L.; Richardson, D.; Butt, J. Biochemistry 2001, 40, 11294-307.

(17) Frangioni, B.; Arnoux, P.; Sabaty, M.; Pignol, D.; Bertrand, P.; Guigliarelli, B.; Léger, C. J. Am. Chem. Soc. 2004, 126, 1328-9.

(18) Hoke, K.; Cobb, N.; Armstrong, F.; Hille, R. Biochemistry 2004, 43, 1667-1674.

(19) Elliott, S. J.; McElhaney, A. E.; Feng, C.; Enemark, J. H.; Armstrong, F. A. J. Am. Chem. Soc. 2002, 124, 11612-3.

(20) Aguey-Zinsou, K.-F.; Bernhardt, P. V.; Kappler, U.; McEwan, A. G. J. Am. Chem. Soc. $2003,125,530-5$.

(21) Ferapontova, E. E.; Ruzgas, T.; Gorton, L. Anal. Chem. 2003, 75, 4841-4850.

(22) Frasca, S.; Rojas, O.; Salewski, J.; Neumann, B.; Stiba, K.; Weidinger, I. M.; Tiersch, B.; Leimkühler, S.; Koetz, J.; Wollenberger, U. Bioelectrochemistry 2012, 87, 33-41.

(23) Zeng, T.; Leimkühler, S.; Koetz, J.; Wollenberger, U. ACS Appl. Mater. Interfaces 2015, 7, 21487-21494. 
(24) Zeng, T.; Frasca, S.; Rumschöttel, J.; Koetz, J.; Leimkühler, S.; Wollenberger, U. Electroanalysis 2016, 28, 2303-2310.

(25) Sezer, M.; Spricigo, R.; Utesch, T.; Millo, D.; Leimkuehler, S.; Mroginski, M. A.; Wollenberger, U.; Hildebrandt, P.; Weidinger, I. M. Phys. Chem. Chem. Phys. 2010, 12, 7894-7903.

(26) Saengdee, P.; Promptmas, C.; Zeng, T.; Leimkühler, S.; Wollenberger, U. Electroanalysis 2017, 29, 110-115.

(27) Zeng, T.; Pankratov, D.; Falk, M.; Leimkühler, S.; Shleev, S.; Wollenberger, U. Biosens Bioelectron 2015, 66, 39-42.

(28) Fourmond, V.; Burlat, B.; Dementin, S.; Arnoux, P.; Sabaty, M.; Boiry, S.; Guigliarelli, B.; Bertrand, P.; Pignol, D.; Léger, C. J. Phys. Chem. B 2008, 112, 15478-15486.

(29) Ceccaldi, P.; Rendon, J.; Léger, C.; Toci, R.; Guigliarelli, B.; Magalon, A.; Grimaldi, S.; Fourmond, V. Biochim. Biophys. Acta 2015, 1847, 1055-1063.

(30) Utesch, T.; Sezer, M.; Weidinger, I. M.; Mroginski, M. A. Langmuir 2012, 28, 5761-5769.

(31) Fourmond, V.; Léger, C. Curr. Opin. Electrochem. 2017, 1, 110-120.

(32) Bertrand, P.; Frangioni, B.; Dementin, S.; Sabaty, M.; Arnoux, P.; Guigliarelli, B.; Pignol, D.; Léger, C. J. Phys. Chem. B 2007, 111, 10300-10311.

(33) Léger, C.; Lederer, F.; Guigliarelli, B.; Bertrand, P. J. Am. Chem. Soc. 2006, 128, 180-187.

(34) Armstrong, F. A.; Camba, R.; Heering, H. A.; Hirst, J.; Jeuken, L. J.; Jones, A. K.; Léger, C.; McEvoy, J. P. Faraday Discuss. 2000, 116, 191-203.

(35) Chen, K.; Hirst, J.; Camba, R.; Bonagura, C. A.; Stout, C. D.; Burgess, B. K.; Armstrong, F. A. Nature 2000, 405, 814-7. 
(36) Jones, A. K.; Camba, R.; Reid, G. A.; Chapman, S. K.; Armstrong, F. A. J. Am. Chem. Soc. 2000, 122, 6494-6495.

(37) Limoges, B.; Saveant, J.-M. J. Electroanal. Chem. 2004, 562, 43-52.

(38) Feng, C.; Wilson, H. L.; Tollin, G.; Astashkin, A. V.; Hazzard, J. T.; Rajagopalan, K. V.; Enemark, J. H. Biochemistry 2005, 44, 13734-13743.

(39) Wilson, H. L.; Wilkinson, S. R.; Rajagopalan, K. V. Biochemistry 2006, 45, 2149-2160.

(40) Limoges and Savéant ${ }^{37}$ had to assume that electron-transfer is infinitely fast to avoid this complication.

(41) Johnson-Winters, K.; Nordstrom, A. R.; Emesh, S.; Astashkin, A. V.; Rajapakshe, A.; Berry, R. E.; Tollin, G.; Enemark, J. H. Biochemistry 2010, 49, 1290-1296.

(42) Feng, C.; Wilson, H. L.; Hurley, J. K.; Hazzard, J. T.; Tollin, G.; Rajagopalan, K. V.; Enemark, J. H. Biochemistry 2003, 42, 12235-12242.

(43) Spence, J. T.; Kipke, C. A.; Enemark, J. H.; Sunde, R. A. Inorg. Chem. 1991, 30, 30113015.

(44) Heering, H. A.; Hirst, J.; Armstrong, F. A. J. Phys. Chem. B 1998, 102, 6889-6902.

(45) Fourmond, V.; Sabaty, M.; Arnoux, P.; Bertrand, P.; Pignol, D.; Léger, C. J. Phys. Chem. B 2010, 114, 3341-3347.

(46) Jacques, J. G.; Burlat, B.; Arnoux, P.; Sabaty, M.; Guigliarelli, B.; Léger, C.; Pignol, D.; Fourmond, V. Biochim. Biophys. Acta 2014, 1837, 1801-1809.

(47) Astashkin, A. V.; Rajapakshe, A.; Cornelison, M. J.; Johnson-Winters, K.; Enemark, J. H. J. Phys. Chem. B 2012, 116, 1942-1950.

(48) Temple, C. A.; Graf, T. N.; Rajagopalan, K. V. Arch. Biochem. Biophys. 2000, 383, 281-287. 
(49) Spricigo, R.; Dronov, R.; Rajagopalan, K. V.; Lisdat, F.; Leimkuhler, S.; Scheller, F. W.; Wollenberger, U. Soft Matter 2008, 4, 972-978.

(50) Note, C.; Kosmella, S.; Koetz, J. Colloids Surf., A 2006, 290, 150-156.

(51) Fourmond, V. Anal. Chem. 2016, 88, 5050-5052.

(52) Bader, G.; Deuflhard, P. Numer. Math 1983, 41, 373-398. 
Graphical TOC Entry

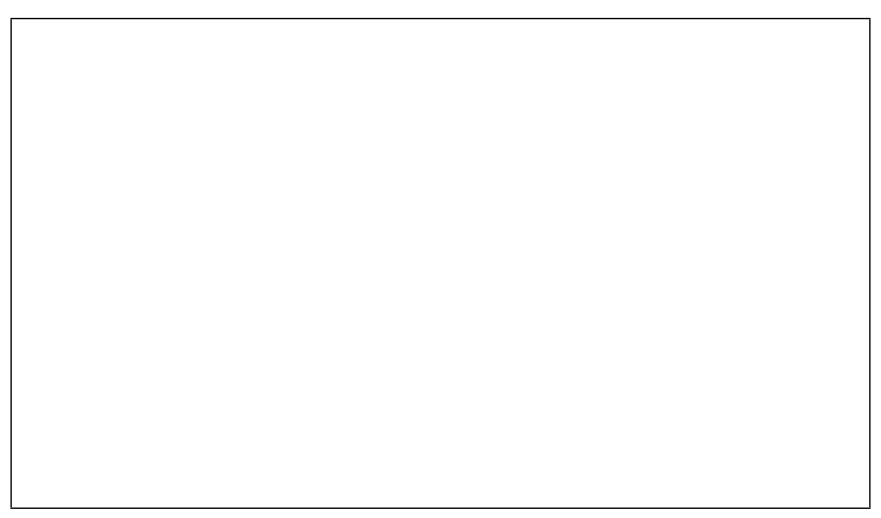

